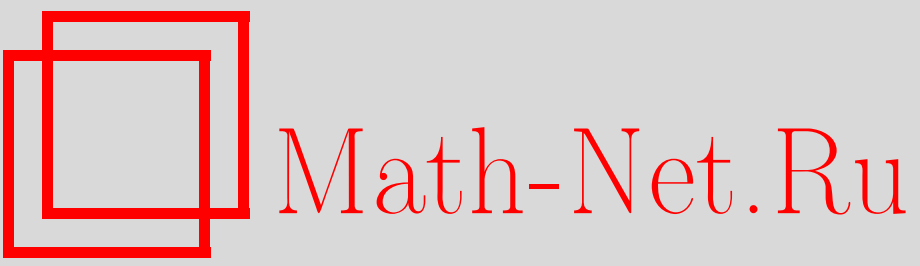

Е. В. Биткина, В. Г. Пидодня, О. В. Биткина, Исследование влияния технологических факторов на остаточные напряжения в волокнистом композите, Beстн. Сам. гос. техн. ун-та. Сер. Физ.-мат. науки, 2011, выпуск 4(), 59-66

DOI: https://doi.org/10.14498/vsgtu961

Использование Общероссийского математического портала Math-Net.Ru подразумевает, что вы прочитали и согласны с пользовательским соглашением

http://www. mathnet.ru/rus/agreement

Параметры загрузки:

IP : 107.22 .136 .117

26 апреля 2023 г., 15:30:50 
УДК 539.373

\title{
ИССЛЕДОВАНИЕ ВЛИЯНИЯ ТЕХНОЛОГИЧЕСКИХ ФАКТОРОВ НА ОСТАТОЧНЫЕ НАПРЯЖЕНИЯ В ВОЛОКНИСТОМ КОМПОЗИТЕ
}

\author{
Е. В. Биткина , В.Г. Пидодня ${ }^{1}$ О.В. Биткина ${ }^{2}$ \\ 1 Сызранский филиал Самарского государственного технического университета, \\ 446001, Самарская обл., Сызрань, ул. Советская, 45 \\ 2 Самарский государственный технический университет, \\ 443100, Самара, ул. Молодогвардейская, 244. \\ E-mails: elena_bitkina@mail.ru; ol_vl_bitkina@mail.ru
}

Разработан метод оченки напряжённо-деформированного состояния композиционных материалов и конструкиий с учётом температурных и технологических воздействий. Изучено влияние обгёмного содержания компонентов композита, величины разности между температурами отверждения и эксплуатации, а также уровня предварительного натяжения волокон на распределение остаточных температурных эквивалентных напряжений в многослойных композитных панелях.

Ключевые слова: композичионные материалы, остаточные напряжения, анизотропия, термомеханические свойства, размеростабилъные конструкции.

Введение. Композиционные материалы находят все более широкое применение в различных отраслях техники, что объясняется широким спектром свойств, выгодно отличающих их от традиционных материалов и сплавов: высокая удельная прочность, жаростойкость, усталостная и длительная прочность и т. Д.

Данный факт объясняет необходимость исследований, направленных на создание новых конструкций из композитов. В частности, большое внимание в настоящее время уделяется проблеме создания размеростабильных крупногабаритных конструкций.

Размеростабильные крупногабаритные конструкции изготавливаются путём послойной укладки препрегов на технологические формы или оправки, определяющие заданную геометрию изделия, с последующим отверждением полуфабриката в автоклаве или печи при повышенной температуре и избыточном давлении $[1,2]$.

Поскольку в результате отверждения слои оказываются жёстко связанными (склеенными), то при охлаждении, после завершения процесса отверждения, в них образуются несвободные температурные деформации, приводящие к технологическим остаточным напряжениям и остаточным деформациям (короблению).

Очевидно, что эти напряжения могут оказывать влияние как на несущую способность элементов конструкции из композиционных материалов, так и на характер их разрушений под действием внешнего нагружения, а остаточные деформации будут определять геометрическую точность изготавливаемого изделия

Расчётная модель многослойных панелей из композиционных материалов. Одним из актуальных вопросов проектирования элементов размероста-

Елена Владимировна Биткина (к.т.н., доц.), доцент, каф. технической механики. Владимир Григоръевич Пидодня (к.т.н., доц.), зав. кафедрой, каф. технической механики. Олъга Владимировна Биткина, аспирант, каф. прикладной математики и информатики. 
бильных конструкций из композиционных материалов является обоснование новых расчетных схем, связанных с особенностями поведения слоистых композитных конструкций с учётом технологических воздействий при их изготовлении.

В данной работе рассматриваются композиционные слоистые панели, изготовленные при температуре отверждения $T$, и находящиеся под воздействием температурного поля.

В целях управления начальным напряжённо-деформированным состоянием панелей, понижения деформативности, повышения несущей способности и геометрической точности конструкции слои препрега предварительно натянуты, после отверждения натяжение снято. Продольные и поперечные кромки панели свободны.

В качестве расчетной модели предлагается схематизация изучаемой тонкостенной пространственной системы в виде анизотропной модели.

Модель напряжённо-деформированного состояния композитной панели. Для изучения напряжённо-деформированного состояния многослойного пакета плоских панелей тонкостенных пространственных конструкций, изготовленных из новых материалов композиционного типа, при построении расчетной схемы должны быть приняты во внимание специфические особенности композитов, в том числе анизотропия термомеханических свойств [3].

В рамках анизотропной модели, пользуясь технической теорией тонких пластинок, рассмотрим расчет многослойной панели, обладающей анизотропией вследствие несимметрии свойств структуры пакета по толщине и находящейся под действием температурного поля $T$ и технологического натяжения $\mathrm{K}_{\mathrm{HB}}$ (рис. 1$)$.

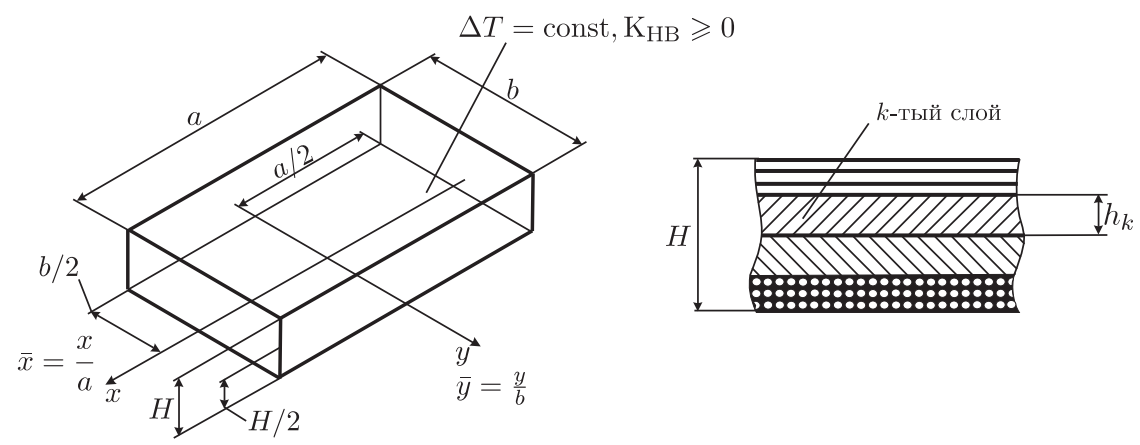

Рис. 1. Геометрическая модель и схема нагружения слоистой панели из композиционных материалов с несимметричной структурой по толщине после отверждения

Препреги предварительно натянуты, после отверждения натяжение снято. Так как структура слоистого элемента несимметрична, при последующей реализации гипотез о нормальном элементе в расчетной схеме теряется смысл срединной поверхности. Поэтому плоскость приведения, в которой располагаются координатные оси, и, соответственно, начало отсчёта координаты $Z$, в дальнейшем могут быть выбраны произвольно. С использованием гипотезы Кирхгофа, соотношений Коши, формул геометрических преобразований, а также закона Гука с учётом влияния температуры и формул преобразования напряжений при повороте осей $[2,4,5]$ получим выражения для компонент состояния $k$-того слоя [5]: 


$$
\begin{gathered}
\left\{\begin{array}{c}
\sigma_{x} \\
\sigma_{y} \\
\tau_{x y}
\end{array}\right\}^{(K)}=\left[\begin{array}{ccc}
\bar{Q}_{11} & \bar{Q}_{12} & \bar{Q}_{16} \\
\bar{Q}_{21} & \bar{Q}_{22} & \bar{Q}_{26} \\
\bar{Q}_{16} & \bar{Q}_{26} & \bar{Q}_{66}
\end{array}\right]^{(K)}\left\{\begin{array}{c}
\varepsilon_{x}^{0}+K_{x}^{0} z^{(K)}-\bar{\alpha}_{1}^{(K)} \Delta T-\bar{\varepsilon}_{1}^{(K)} \\
\varepsilon_{y}^{0}+K_{y}^{0} z^{(K)}-\bar{\alpha}_{2}^{(K)} \Delta T-\bar{\varepsilon}_{2}^{(K)} \\
\gamma_{x y}^{0}+K_{x y}^{0} z^{(K)} \bar{\alpha}_{6}^{(K)} \Delta T-\bar{\varepsilon}_{6}^{(K)}
\end{array}\right\}, \\
\varepsilon_{x}=\varepsilon_{x}^{0}+K_{x}^{0} z=\frac{\partial U_{0}}{\partial x}-\frac{\partial^{2} W}{\partial x^{2}} z ; \quad \varepsilon_{y}=\varepsilon_{y}^{0}+K_{y}^{0} z=\frac{\partial V_{0}}{\partial y}-\frac{\partial^{2} W}{\partial y^{2}} z ; \\
\gamma_{x y}=\gamma_{x y}^{0}+K_{x y}^{0} z=\frac{\partial U_{0}}{\partial y}+\frac{\partial V_{0}}{\partial x}-2 \frac{\partial^{2} W}{\partial x \partial y} z
\end{gathered}
$$

где $\varepsilon_{x}^{0}, \varepsilon_{y}^{0}, \gamma_{x y}^{0}$ - деформации в плоскости приведения; $K_{x}^{0}, K_{y}^{0}, K_{x y}^{0}-$ кривизны панели.

Жёсткости слоя $\bar{Q}_{I J}^{(K)}(I, J=1,2,6)$, коэффициенты температурного расширения $\bar{\alpha}_{J}^{(K)}$ и деформации натяжения слоя $\bar{\varepsilon}_{H J}^{(K)}$ в $(1)$, приведённые к осям панели, определяются через жёсткостные и температурные характеристики слоя, заданные в системе координат $(1,2)$, с ним связанной, с учётом поворота последней относительно декартовой системы координат пластинки $(x, y)$. Соответствующие преобразования для $\bar{Q}_{I J}^{(K)}$ и $\bar{\alpha}_{J}^{(K)}$ представлены в работах $[2,4,5]$. Жёсткости слоя, приведенные к осям панели, определяются соотношением

$$
\left\{\begin{array}{l}
\bar{Q}_{11} \\
\bar{Q}_{12} \\
\bar{Q}_{22} \\
\bar{Q}_{66} \\
\bar{Q}_{16} \\
\bar{Q}_{26}
\end{array}\right\}=\left[\begin{array}{cccc}
m^{4} & 2 m^{2} n^{2} & n^{4} & 4 m^{2} n^{2} \\
m^{2} n^{2} & m^{4}+n^{4} & m^{2} n^{2} & -4 m^{2} n^{2} \\
n^{4} & 2 m^{2} n^{2} & m^{4} & 4 m^{2} n^{2} \\
m^{2} n^{2} & -2 m^{2} n^{2} & m^{2} n^{2} & \left(m^{2}-n^{2}\right)^{2} \\
m^{3} n & -m n\left(m^{2}-n^{2}\right) & -m n^{3} & -2 m n\left(m^{2}-n^{2}\right) \\
m n^{3} & m n\left(m^{2}-n^{2}\right) & -m^{3} n & 2 m n\left(m^{2}-n^{2}\right)
\end{array}\right]^{(K)}\left\{\begin{array}{c}
Q_{11} \\
Q_{12} \\
Q_{22} \\
Q_{66}
\end{array}\right\}
$$

где

$$
\begin{aligned}
& Q_{11}^{(K)}=\frac{E_{1}^{(K)}}{1-\nu_{12}^{(K)} \nu_{21}^{(K)}} ; \quad Q_{12}^{(K)}=Q_{21}^{(K)}=Q_{11}^{(K)} \nu_{21}^{(K)} ; \\
& Q_{22}^{(K)}=\frac{E_{2}^{(K)}}{1-\nu_{12}^{(K)} \nu_{21}^{(K)}} ; \quad Q_{66}^{(K)}=G_{12}^{(K)} .
\end{aligned}
$$

Аналогичное преобразование для коэффициентов температурного расширения имеет следующий вид:

$$
\left\{\begin{array}{c}
\bar{\alpha}_{1} \\
\bar{\alpha}_{2} \\
\bar{\alpha}_{6}
\end{array}\right\}^{(K)}=\left[\begin{array}{cc}
m^{2} & n^{2} \\
n^{2} & m^{2} \\
2 m n & -2 m n
\end{array}\right]^{(K)}\left\{\begin{array}{l}
\alpha_{1} \\
\alpha_{2}
\end{array}\right\}^{(K)} .
$$

Для деформации натяжения имеем

$$
\left\{\begin{array}{l}
\bar{\varepsilon}_{H 1} \\
\bar{\varepsilon}_{H 2} \\
\bar{\varepsilon}_{H 6}
\end{array}\right\}^{(K)}=\left\{\begin{array}{c}
m^{2} \\
n^{2} \\
2 m n
\end{array}\right\}^{(K)}\left\{\varepsilon_{H}\right\}^{(K)},
$$


где $m^{(K)}$ и $n^{(K)}$ - тригонометрические функции угла поворота осей координат $(1,2)$ связанных с $k$-тым слоем, относительно декартовой системы координат $(x, y)$, а именно: $m^{(K)}=\cos \varphi_{K}, n^{(K)}=\sin \varphi_{K}$, а $\bar{\varepsilon}_{H}^{(K)}=K_{\mathrm{HB}} \bar{\varepsilon}_{\mathrm{B}}^{(K)}$, где $\mathrm{K}_{\mathrm{HB}}$ - безразмерный коэффициент, определяющий уровень натяжения и изменяющийся (по модулю) от 0 до $1 ; \bar{\varepsilon}_{\mathrm{B}}^{(K)}$ - допускаемая деформация препрега $k$-того слоя.

Модули упругости в направлении слоя $E_{1}^{(K)}$ и перпендикулярного к нему $E_{2}^{(K)}$, соответственно, модуль сдвига $G_{12}^{(K)}$, коэффициенты Пуассона $\nu_{12}^{(K)}, \nu_{21}^{(K)}$ и коэффициенты температурного расширения $\alpha_{1}^{(K)}$ и $\alpha_{2}^{(K)}$ зависят от температурных свойств волокон и матрицы, а также от степени армирования слоя.

При записи физических соотношений в виде (1) не учитывается, что в препреге (слое) предварительно натягиваются лишь волокна, в то время как после снятия натяжения слой деформируется полностью.

Погонные нормальные и сдвигающие силы, изгибающие и крутящие моменты для всего пакета, действующие, например, по площадке, перпендикулярной оси $x$, определяются интегрированием соответствующих компонент напряжённого состояния по толщине, что с учётом гипотезы Кирхгофа даёт:

$$
\begin{array}{cc}
N_{x}=\int_{-H / 2}^{H / 2} \sigma_{x}^{(K)} d z ; & N_{x y}=\int_{-H / 2}^{H / 2} \tau_{x y}^{(K)} d z ; \\
M_{x}=\int_{-H / 2}^{H / 2} \sigma_{x}^{(K)} z d z ; & H_{x y}=\int_{-H / 2}^{H / 2} \tau_{x y}^{(K)} z d z .
\end{array}
$$

Отсюда и из (1) вытекают соотношения, связывающие внутренние силовые факторы с деформацией слоистого элемента:

$$
\left\{\begin{array}{c}
N_{I} \\
N_{J}
\end{array}\right\}=\left[\begin{array}{ll}
A_{I J} & B_{I J} \\
B_{I J} & D_{I J}
\end{array}\right]\left\{\begin{array}{c}
\varepsilon_{J}^{0} \\
K_{J}^{0}
\end{array}\right\}-\left\{\begin{array}{c}
N_{I}^{T} \\
M_{I}^{T}
\end{array}\right\}-\left\{\begin{array}{c}
N_{I}^{H} \\
M_{I}^{H}
\end{array}\right\}_{\substack{I, J=1,2,6 \\
(x, y, x y)}},
$$

где $N_{x}^{T}, N_{y}^{T}, N_{x y}^{T}, M_{x}^{T}, M_{Y}^{T}, H_{x y}^{T}$ - «температурные» усилия и моменты.

$$
\begin{gathered}
\left\{N_{I}^{T}\right\}=\Delta T \sum_{k=1}^{N}\left[Q_{I J}\right]^{(K)}\left\{\alpha_{J}\right\}^{(K)} F^{(K)} ; \\
\left\{M_{I}^{T}\right\}=\Delta T \sum_{k=1}^{N}\left[Q_{I J}\right]^{(K)}\left\{\alpha_{J}\right\}^{(K)} S_{\substack{I, J=1,2,6 \\
(x, y, x y)}}^{(K)}
\end{gathered}
$$

a $N_{x}^{H}, N_{y}^{H}, N_{x y}^{H}, M_{x}^{H}, M_{y}^{H}, H_{x y}^{H}$ - усилия и моменты от натяжения:

$$
\begin{gathered}
\left\{N_{I}^{H}\right\}=\sum_{k=1}^{N}\left[Q_{I J}\right]^{(K)}\left\{\varepsilon_{I J}\right\}^{(K)} F^{(K)} ; \\
\left\{M_{I}^{H}\right\}=\sum_{k=1}^{N}\left[Q_{I J}\right]^{(K)}\left\{\varepsilon_{I J}\right\}^{(K)} S_{\substack{I, J=1,2,6 \\
(x, y, x y)}}^{(K)}
\end{gathered}
$$

В свою очередь, $F^{(K)}, S^{(K)}, J^{(K)}$ - погонные площадь, статический момент и момент инерции $k$-того слоя соответственно.

Обобщённые жёсткостные характеристики многослойной панели $\left(A_{I J}, B_{I J}\right.$, $\left.D_{I J}\right)$ даны в работах $[4,5,6]$. 
Так как усилия и моменты согласно формулам (2) зависят как от деформации в плоскости панели, так и от кривизны поверхности приведения, в рассматриваемом случае из-за несимметрии свойств структуры пакета по толщине задача не разделяется на плоскую задачу и задачу об изгибе пластинки.

Если известен столбец внутренних силовых факторов, в том числе температурных и связанных с натяжением, на основании равенства (2) путём обращения матриц могут быть найдены компоненты деформированного состояния:

$$
\left\{\begin{array}{l}
\varepsilon_{J}^{0} \\
K_{J}^{0}
\end{array}\right\}=\left[\begin{array}{ll}
A_{I J} & B_{I J} \\
B_{I J} & D_{I J}
\end{array}\right]^{-1}\left\{\begin{array}{c}
N_{I}^{T}+N_{I}^{H} \\
M_{I}^{T}+M_{I}^{H}
\end{array}\right\}
$$

а следовательно, и напряжения по формуле (1):

$$
\left\{\begin{array}{c}
\sigma_{x} \\
\sigma_{y} \\
\tau_{x y}
\end{array}\right\}^{(K)}=\left[\begin{array}{lll}
\bar{Q}_{11} & \bar{Q}_{12} & \bar{Q}_{16} \\
\bar{Q}_{12} & \bar{Q}_{22} & \bar{Q}_{26} \\
\bar{Q}_{16} & \bar{Q}_{26} & \bar{Q}_{66}
\end{array}\right]^{(K)}\left\{\begin{array}{c}
\varepsilon_{x}^{0}+K_{x}^{0} z-\bar{\alpha}_{1}^{(K)} \Delta T-\bar{\varepsilon}_{H_{1}}^{(K)} \\
\varepsilon_{y}^{0}+K_{y}^{0} z-\bar{\alpha}_{2}^{(K)} \Delta T-\bar{\varepsilon}_{H_{2}}^{(K)} \\
\gamma_{x y}^{0}+K_{x y}^{0} z-\bar{\alpha}_{6}^{(K)} \Delta T-\bar{\varepsilon}_{H_{6}}^{(K)}
\end{array}\right\} .
$$

В системе координат, связанной с направлением армирования $k$-того слоя, напряжённое состояние определяется посредством преобразования при повороте осей:

$$
\left\{\begin{array}{l}
\sigma_{1} \\
\sigma_{2} \\
\sigma_{3}
\end{array}\right\}^{(K)}=\left[\begin{array}{ccc}
m^{2} & n^{2} & 2 m n \\
n^{2} & m^{2} & -2 m n \\
-m n & m n & \left(m^{2}-n^{2}\right)
\end{array}\right]^{(K)}\left\{\begin{array}{c}
\sigma_{x} \\
\sigma_{y} \\
\tau_{x y}
\end{array}\right\} .
$$

Согласно критерию прочности Гольденблата-Копнова для однонаправленного слоя композиционного материала растрескивания или разрушения пакета не произойдёт, пока в каждом слое выполняется следующее неравенство:

$$
\begin{aligned}
{\left[\frac{1}{4}\left(\frac{1}{\bar{\sigma}_{1 \mathrm{p}}^{(K)}}+\frac{1}{\bar{\sigma}_{1 \mathrm{c}}^{(K)}}\right)^{2}\left(\sigma_{1}^{(K)}\right)^{2}+\frac{1}{4}\left(\frac{1}{\bar{\sigma}_{2 \mathrm{p}}^{(K)}}+\frac{1}{\bar{\sigma}_{2 \mathrm{c}}^{(K)}}\right)^{2}\left(\sigma_{2}^{(K)}\right)^{2}+\left(\frac{\tau_{12}^{(K)}}{\bar{\tau}_{12}^{(K)}}\right)^{2}\right]^{\frac{1}{2}}+} \\
+\frac{1}{2}\left(\frac{1}{\bar{\sigma}_{1 \mathrm{p}}^{(K)}}+\frac{1}{\bar{\sigma}_{1 \mathrm{c}}^{(K)}}\right)^{2} \sigma_{1}^{(K)}+\frac{1}{2}\left(\frac{1}{\bar{\sigma}_{2 \mathrm{p}}^{(K)}}+\frac{1}{\bar{\sigma}_{2 \mathrm{c}}^{(K)}}\right)^{2} \sigma_{2}^{(K)}=\sigma_{\text {эКв }} \leqslant 1
\end{aligned}
$$

где $\bar{\sigma}_{1 \mathrm{p}}^{(K)}, \bar{\sigma}_{1 \mathrm{c}}^{(K)}, \bar{\sigma}_{2 \mathrm{p}}^{(K)}, \bar{\sigma}_{2 \mathrm{c}}^{(K)}, \bar{\tau}_{12}^{(K)}$ - допускаемые напряжения с индексом р - на растяжение, с индексом с - на сжатие соответственно, имеющие такой вид:

$$
\begin{gathered}
\bar{\sigma}_{1 \mathrm{p}}^{(K)}=\bar{\sigma}_{\mathrm{B}}^{(K)} V_{\mathrm{B}}+\bar{\sigma}_{\mathrm{M}}^{(K)} V_{\mathrm{M}}^{(K)}, \quad \bar{\sigma}_{1 \mathrm{c}}^{(K)}=2 V_{\mathrm{B}}^{(K)}\left[\frac{V_{\mathrm{B}}^{(K)} E_{\mathrm{M}}^{(K)}}{V_{\mathrm{M}}^{(K)}}\right]^{\frac{1}{2}}, \\
\bar{\sigma}_{2 \mathrm{p}}^{(K)}=0,5 \bar{\sigma}_{\mathrm{M}}^{(K)}, \quad \bar{\sigma}_{2 \mathrm{c}}^{(K)}=0,5 \bar{\sigma}_{\mathrm{M}}^{(K)}, \quad \bar{\tau}_{12}^{(K)}=0,5 \bar{\tau}_{\mathrm{M}}^{(K)} .
\end{gathered}
$$

Здесь индексами В и М обозначены термоупругие характеристики волокна и матрицы соответственно.

Формоизменение, или коробление, изготавливаемых конструкций определяется рядом факторов, которые следует учитывать как при проектировании, так при изготовлении. 
Используя полученные выше соотношения и интегрируя выражения

$$
U=U_{0}(x, y)-\frac{\partial W}{\partial x} z ; \quad V=V_{0}(x, y)-\frac{\partial W}{\partial y} z ; \quad W=W(x, y)
$$

определяющие компоненты кривизны панели, получим, с точностью до произвольных постоянных, формулы для прогиба $W$, а также для продольного $U_{0}$ и тангенциального $V_{0}$ смещений:

$$
W=-\frac{1}{2}\left(K_{x}^{0} x^{2}+K_{x y}^{0} x y+K_{y}^{0} y^{2}\right) ; \quad U_{0}=\varepsilon_{x}^{0} x+\frac{\gamma_{x y}^{0}}{2} y ; \quad V_{0}=\varepsilon_{y}^{0} y+\frac{\gamma_{x y}^{0}}{2} x .
$$

В соответствии с приведенными формулами было исследовано влияние предварительного натяжения волокон и радиуса волокна на распределение остаточных эквивалентных напряжений в слоях восьмислойной углепластиковой панели с продольно-поперечной укладкой $\left(0_{4}^{\circ} / 90_{4}^{\circ}\right)$ при автоклавном методе изготовления (температура отверждения составляет $170{ }^{\circ} \mathrm{C}$, разница между начальной температурой и температурой отверждения $\Delta T=150^{\circ} \mathrm{C}$.

Сравнение результатов распределения эквивалентных остаточных технологических напряжений $\sigma_{\text {экв }}$ в процессе охлаждения при разных уровнях предварительного натяжения волокон $\mathrm{K}_{\mathrm{HB}}$ позволяет установить оптимальную величину степени армирования слоя $V_{\mathrm{B}}$ с целью обеспечения изготовления изделий с монолитной структурой. Некоторые результаты исследования частично приведены на рис. $2,3$.

Анализ распределения эквивалентных $\sigma_{\text {экв }}$ остаточных напряжений в слоях восьмислойной углепластиковой панели с продольно-поперечной укладкой $\left(0_{4}^{\circ} / 90_{4}^{\circ}\right)$ в процессе охлаждения при степени армирования слоя $V_{\mathrm{B}}=0,5,0,65$ и 0,8 и уровня натяжения $\mathrm{K}_{\mathrm{HB}}=0,4$ показывает, что напряжения при охлаждении пластин до температуры эксплуатации, например, $T=20^{\circ} \mathrm{C}(\Delta T=$ $=-150^{\circ} \mathrm{C}$ ) меняют свой знак на противоположный. Величина натяжения

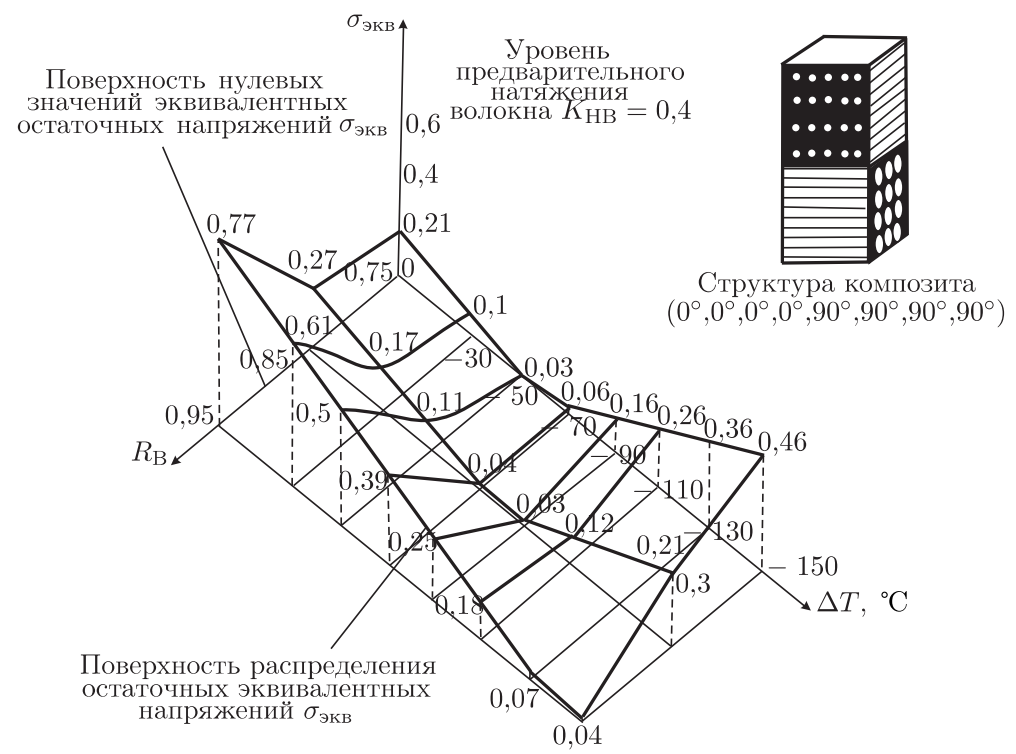

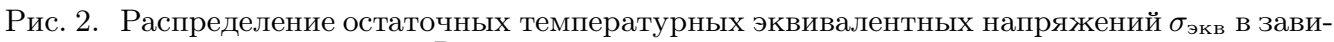
симости от радиуса волокна $R_{\mathrm{B}}$ и величины разности между температурами отверждения и эксплуатации $\Delta T$ при уровне предварительного натяжения волокон $\mathrm{K}_{\mathrm{HB}}=0,4$ 


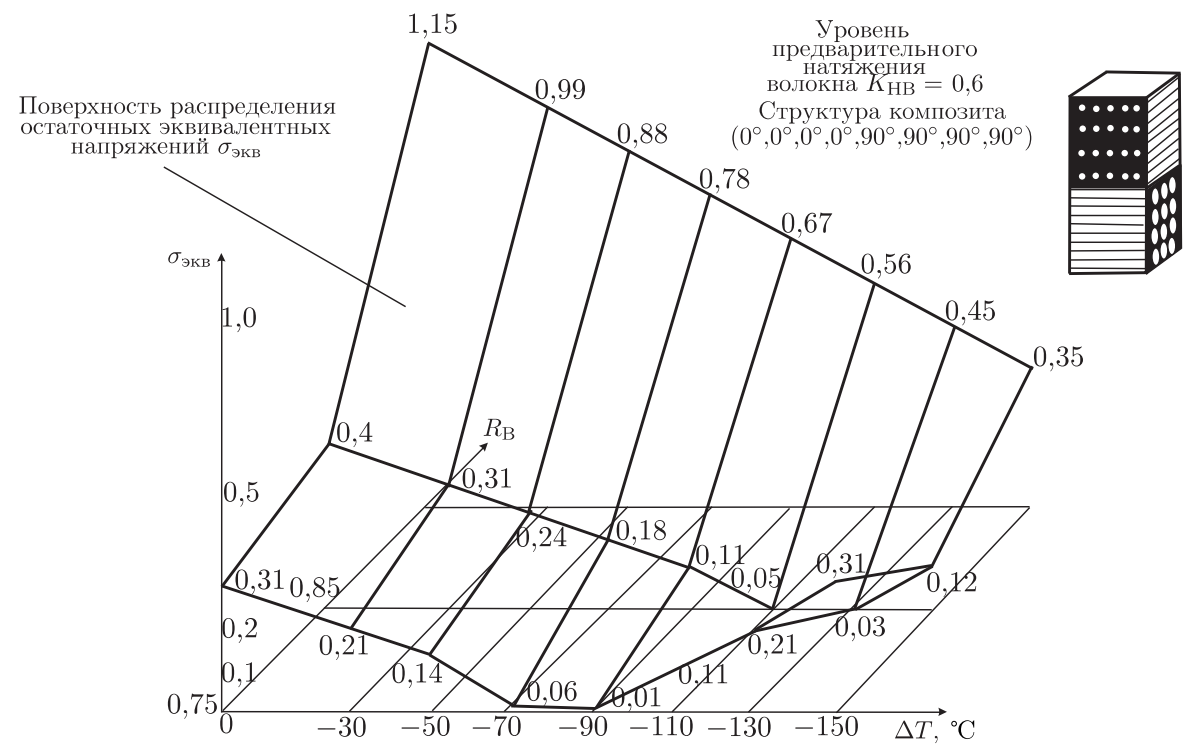

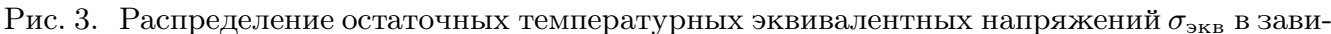
симости от радиуса волокна $R_{\mathrm{B}}$ и величины разности между температурами отверждения и эксплуатации $\Delta T$ при уровне предварительного натяжения волокон $\mathrm{K}_{\mathrm{HB}}=0,6$

$\mathrm{K}_{\mathrm{HB}}=0,4$ наиболее эффективна для степени армирования слоя $V_{\mathrm{B}}=0,8$ (см. рис. 2).

Следует отметить, что для каждой степени армирования слоя $V_{\mathrm{B}}$ существуют строго определенные границы интервала величины натяжения $\mathrm{K}_{\mathrm{HB}}$. Так, для степени армирования слоя $V_{\mathrm{B}}=0,8$ уровень предварительного натяжения находится в пределах $0 \leqslant \mathrm{~K}_{\mathrm{HB}} \leqslant 0,42$. Увеличение значения данного уровня $\mathrm{K}_{\mathrm{HB}}$ приводит к растрескиванию слоев уже на начальном этапе охлаждения. В то же время уровень предварительного натяжения волокон $\mathrm{K}_{\mathrm{HB}}=0,4$ позволяет создавать практически ненапряженную конструкцию, т. е. $\sigma_{\text {экв }}=0$. Более высокий уровень натяжения $\mathrm{K}_{\mathrm{HB}}=0,8$ наиболее эффективен для степени армирования слоя $V_{\mathrm{B}}=0,65$, когда технологические напряжения становятся минимальными. Для степеней армирования $V_{\mathrm{B}}$, изменяющихся в пределах от 0,5 до 0,65, величина натяжения $\mathrm{K}_{\mathrm{HB}}$ может меняться в интервале от 0 до 0,8. Следует отметить, что для получения минимальных остаточных напряжений более высокая степень армирования слоя $V_{\mathrm{B}}$ должна соответствовать меньшему значению уровня предварительного натяжения $\mathrm{K}_{\mathrm{HB}}$.

Таким образом, анализ оценки влияния технологического натяжения слоев с учётом степени армирования слоя на уровень остаточных напряжений в слоистых панелях, приведенный выше, позволяет устанавливать величины натяжения, обеспечивающие монолитность структуры слоистых элементов конструкции при их изготовлении.

\section{БИБЛИОГРАФИЧЕСКИЙ СПИСОК}

1. Молодиов Г.А., Биткин В.Е., Симонов В.Ф., Урмансов Ф.Ф. Формостабильные и интеллектуальные конструкции из композиционных материалов. М.: Машиностроение, 2000. 352 c. [Molodtsov G. A., Bitkin V.E., Simonov V.F., Urmansov F. F. Stable-shape and intelligent structures of composite materials. Moscow: Mashinostroenie, 2000. 352 pp.]

2. Образцов И. Ф., Васильев В. В., Бунаков В. А. Оптимальное армирование оболочек вра- 
щения из композиционных материалов. М.: Машиностроение, 1977. 144 с. [Obraztsov I. F., Vasil'ev V.V., Bunakov V.A. Optimal reinforcement for rotation shells of composite materials. Moscow: Mashinostroenie, 1977. 144 pp.]

3. Биткина E. В. Разработка метода анализа напряжённо-деформированного состояния многослойных композиционных материалов и конструкций с учётом температурных, силовых и технологических воздействий: Дис. ... канд. техн. наук. Самара, 2009. 162 с. [Bitkina E. V. Development of a method of analysis of the stress-strain state of multilayer composite materials and structures subject to temperature, power and technological influences: Ph. D. Thesis (Techn.). Samara, 2009. 162 pp.]

4. Молодиов Г. А. Остаточные напряжения в слоистых анизотропных пластинках / В сб.: Механика композииионных материалов. Вып. 4. М., 1979. С. 730-733. [Molodtsov G. A. Residual stresses in laminated anisotropic plates / In: Mechanics of Composite Materials. Issue 4. Moscow, 1979. Pp. 730-733].

5. Молодиов Г.А., Гавва Л. М., Осинская Е. А. Параметрический анализ остаточных температурных напряжений в плоских слоистых панелях из композиционных материалов. М.: МАИ, 1985. 47 c. [Molodtsov G. A., Gavva L. M., Osinskaya E. A. Parametric analysis of residual thermal stresses in a layered flat panels from composite materials. Moscow: MAI, 1985. 47 pp.]

6. Композиционные материалы. Т. 8: Анализ и проектирование конструкций / ред. К. Чамис. М.: Машиностроение, 1978. 300 с. [ Composite Materials. Vol. Analysis and design of structures / ed. K. Chamis. Moscow: Mashinostroenie, 1978. 300 pp.]

Поступила в редакцию 10/V/2011;

в окончательном варианте - 24/XI/2011.

MSC: 74A10; 74E30

INVESTIGATION OF THE EFFECT OF TECHNOLOGICAL FACTORS ON THE RESIDUAL STRESSES IN FIBROUS COMPOSITES

\author{
E. V. Bitkina ${ }^{1}$, V.G. Pidodnya ${ }^{1}$, O. V. Bitkina ${ }^{2}$ \\ 1 Syzran' Branch of Samara State Technical University, \\ 45, Sovetskaya st., Syzran', Samara region, 446001, Russia. \\ 2 Samara State Technical University, \\ 244, Molodogvardeyskaya st., Samara, 443100, Russia. \\ E-mails: elena_bitkina@mail.ru; ol_vl_bitkina@mail.ru
}

The method of analysis for stress state of the composite materials and structures subject to thermal and technological influences is considered. The influence of the volume content of composite material's components, the magnitude of the difference between curing temperature and operating temperature, as well as the level of fibers tension on the distribution of residual thermal equivalent stresses in multilayered composite panels is explored.

Key words: composite materials, residual stress, anisotropy, thermo-mechanical properties, dimensional stable structures.

Original article submitted $10 / \mathrm{V} / 2011$; revision submitted $24 / \mathrm{XI} / 2011$.

Elena V. Bitkina (Ph. D. (Techn.)), Associate Professor, Dept. of Technical Mechanics. Vladimir G. Pidodnya (Ph. D. (Techn.)), Head of Dept., Dept. of Technical Mechanics. Olga V. Bitkina, Postgraduate Student, Dept. of Applied Mathematics \& Computer Science. 